\title{
ANGULAR MOMENTUM TRANSFER DUE TO GALACTIC WINDS AND COOLING FLOWS
}

\author{
V. MISSOULIS \\ Astronomical Institute, National Observatory of Athens \\ P.O.Box 20048 - GR 11810 Athens - Greece
}

We examine a model of galaxy formation where the bulge is formed at very early stages and this burst of star formation leads to a galactic wind which interacts with a huge surrounding gaseous envelope.

The numerical solutions of the spherically symmetric situation (Missoulis 1994 and references therein) indicate that if the surrounding envelope is constituted mainly of cold clouds, the clouds will evaporate and their matter will be mixed with the hot and metal-enriched gas of the wind. If the surrounding is constituted mainly of diffuse gas, a cold shell will be formed and cloud and star formation are possible.

In a rotating system these phase transitions will lead to angular momentum transfer inwards: In the first case the earlier layers of the wind tend to evaporate clouds from the inner regions. The latter layers tend to evaporate clouds from the outer regions. When the cooling flow starts, the earlier layers with small specific angular momentum are located outside the latter layers. In the second case the angular momentum is transferred when the newly formed clouds from the outer regions start falling towards the center of the system and evaporate at the inner regions. The angular momentum transfer is significant only if the eccentricities of the trajectories of the clouds are large (for the present situation $e \simeq 1$ is expected).

It is clear that in both cases the central regions acquire large specific angular momentum and this is a possible explanation of the origin of Freeman's type II spiral galaxies.

Acknowledgements: The author is grateful to the LOC for financial support.

\section{References}

Missoulis, V. 1994, Astron. Reports, 38, 12 